\title{
Erratum: scalar multiplet dark matter
}

\author{
T. Hambye, ${ }^{a}$ F.-S. Ling, ${ }^{a}$ L. Lopez Honorez ${ }^{a, b}$ and J. Rocher ${ }^{a}$ \\ ${ }^{a}$ Service de Physique Théorique, Université Libre de Bruxelles, \\ 1050 Brussels, Belgium \\ ${ }^{b}$ Departamento de Fúsica Teórica, Universidad Autónoma de Madrid, \\ IFT-UAM/CSIC, 28049 Cantoblanco, Madrid, Spain \\ E-mail: thambye@ulb.ac.be, fling@ulb.ac.be, llopezho@ulb.ac.be, \\ jrocher@ulb.ac. be
}

ERRATUM TO: JHEP07(2009)090

In the paper JHEP07(2009)090 affiliation $b$ should read as:

Departamento de Física Teórica, Universidad Autónoma de Madrid, IFT-UAM/CSIC, 28049 Cantoblanco, Madrid, Spain 